\title{
Realization of Quantum Walks with Negligible Decoherence in Waveguide Lattices
}

\author{
Hagai B. Perets, ${ }^{1, *}$ Yoav Lahini, ${ }^{1}$ Francesca Pozzi, ${ }^{2}$ Marc Sorel, ${ }^{2}$ Roberto Morandotti, ${ }^{3}$ and Yaron Silberberg ${ }^{1}$ \\ ${ }^{1}$ Faculty of Physics, The Weizmann Institute of Science, 76100 Rehovot, Israel \\ ${ }^{2}$ Department of Electronics \& Electrical Engineering, University of Glasgow, Glasgow G12 8QQ, Scotland, United Kingdom \\ ${ }^{3}$ Institute National de la Recherche Scientifique, Universitá du Quebec, Varennes, Quebec J3X 1S2, Canada
}

(Received 24 September 2007; published 2 May 2008)

\begin{abstract}
Quantum random walks are the quantum counterpart of classical random walks, and were recently studied in the context of quantum computation. Physical implementations of quantum walks have only been made in very small scale systems severely limited by decoherence. Here we show that the propagation of photons in waveguide lattices, which have been studied extensively in recent years, are essentially an implementation of quantum walks. Since waveguide lattices are easily constructed at large scales and display negligible decoherence, they can serve as an ideal and versatile experimental playground for the study of quantum walks and quantum algorithms. We experimentally observe quantum walks in large systems ( $\sim 100$ sites) and confirm quantum walks effects which were studied theoretically, including ballistic propagation, disorder, and boundary related effects.
\end{abstract}

DOI: 10.1103/PhysRevLett.100.170506

In classical random walks, a particle starting from an initial site on a lattice randomly chooses a direction, and then moves to a neighboring site accordingly. This process is repeated until some chosen final time. This simple random walk scheme is known to be described by a Gaussian probability distribution of the particle position, where the average absolute distance of the particle from the origin grows as the square root of time. First suggested by Feynman [1] the term quantum random walks was defined to describe the random walk behavior of a quantum particle. The coherent character of the quantum particle plays a major role in its dynamics, giving rise to markedly different behavior of quantum walks (QWs) compared with classical ones. For example, in periodic systems, the quantum particle propagates much faster than its classical counterpart, and its distance from the origin grows linearly with time (ballistic propagation) rather then diffusively [2]. In disordered systems, the expansion of the quantum mechanical wave-function can be exponentially suppressed even for infinitesimal amount of disorder, while such suppression does not occur in classical random walks.

In recent years QWs have been extensively studied theoretically [2] and have been used to devise new quantum computation algorithms [3]. Both discrete and continuous time QWs (DQWs; CQWs) [4-6] have been studied. In DQWs the quantum particle hops between lattice sites in discrete time steps, while in CQW the probability amplitude of the particle leaks continuously to neighboring sites. Experimentally, many methods have been suggested for the implementation of DQWs (see [2]), but only a small scale system consisting of a few states was implemented, using linear optical elements [7]. For CQWs, a few suggestions have been made [8,9], yet only one experimental method have been implemented by realizing a small scale cyclic system (4 states) using a nuclear magnetic resonance system [10]. Such systems are difficult
PACS numbers: 03.67.Lx, 05.40.Fb, 42.25.Dd, 42.50.Xa

to scale to much larger configurations. Moreover, even at these very small scales, errors attributed to decoherence have been observed.

Here we suggest a very different implementation of CQWs using optical waveguide lattices. These systems have been studied extensively in recent years [11], but not in the context of QWs and quantum algorithms. We show that these systems can serve as a unique and robust tool for the study of CQWs. For this purpose we demonstrate three fundamental QW effects that have been theoretically analyzed in the QW literature. These include ballistic propagation in the largest system reported to date ( $\sim 100$ sites), the effects of disorder on QWs, and QWs with reflecting boundary conditions (related to Berry's "particle in a box" and quantum carpets $[12,13])$. Waveguide lattices can be easily realized with even larger scales than shown here $\left(10^{2}-10^{4}\right.$ sites with current fabrication technologies), with practically no decoherence. The high level of engineering and control of these systems enable the study of a wide range of different parameters and initial conditions. Specifically it allows the implementation and study of a large variety of CQWs and show experimental observations of their unique behavior.

The CQW model was first suggested by Farhi and Gutmann [6], where the intuition behind it comes from continuous time classical Markov chains. In the classical random walk on a graph, a step can be described by a matrix $M$ which transforms the probability distribution for the particle position over the graph nodes (sites). The entries of the matrix $M_{j, k}$ give the probability to go from site $j$ to site $k$ in one step of the walk. The idea was to carry this construction over to the quantum case, where the Hamiltonian of the process is used as the generator matrix. The system is evolved using $U(t)=\exp (-i H t)$. If we start in some initial state $\left|\Psi_{\text {in }}\right\rangle$, evolve it under $U$ for a time $T$ and measure the positions of the resulting state, we obtain a 
probability distribution over the vertices of the graph. This is described by

$$
i \frac{\partial \psi_{j}}{\partial t}=-d_{j} \gamma \psi_{j}+\gamma_{j, j+1} \psi_{j+1}+\gamma_{j, j-1} \psi_{j-1},
$$

where $\psi_{j}$ is the wave function at site $j, d_{j}$ is the number of sites connected to site $j\left(d_{j}=2\right.$ in the 1D nearest neighbor case), and $\gamma_{i, j}\left(=\gamma_{j, i}\right)$ is the probability per unit time for the transition between neighboring [14,15]. This mathematical formulation is effectively identical to the well known discrete Schrödinger equation used in the tightbinding (Bloch ansatz) formalism in solid state physics [15]. It is used to describe the evolution of a wave function on a periodic potential, which is essentially the propagation of a quantum particle on a lattice $[16,17]$.

An immediate implication for the correspondence between QWs and these processes is that many of the experiments in solid state physics described by the tight-binding model could serve as implementations of QWs. However, such experiments deal with the macro-physics of the system and with overall observables such as conductance or transmission. Therefore, one cannot measure the specific spatial and temporal distribution of the electrons or photons wave functions and the microphysics of the system cannot be directly observed. Moreover, solid state systems contain many electrons which interact nontrivially and thus cannot be described by the evolution equation of a single particle usually studied in QWs. Consequently, a qualitatively different experimental approach is needed in order to study QWs. Here we report such an approach using waveguide lattices.

Recently, a new technique has been developed for the experimental investigation of periodic systems using optics. The salient feature of these experiments is that evolution of waves in time is also spread out in space, making it much easier to observe. This is achieved by using waveguide structures which are periodic on one dimension [ $x$ axis; see Fig. 1(a)], but are homogeneous along the other ( $z$ axis). In this way the wave propagation along the $z$ axis is free and corresponds to the evolution in time [11]. Under appropriate conditions light is guided inside the waveguides and can coherently tunnel between them. The ex-

(a)

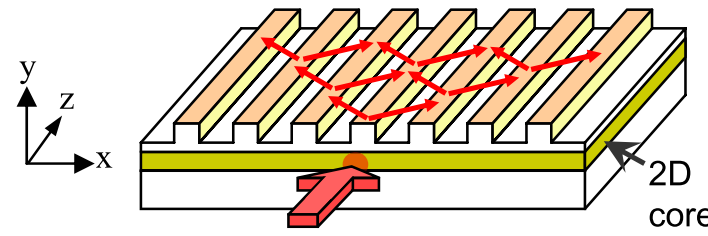

(b)

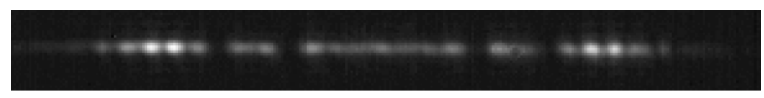

FIG. 1 (color online). (a) Schematic view of the optical waveguide lattice used in the experiments (see text). (b) Image of the output light distribution as recorded in the infrared camera, when the light is injected to a single lattice site at the input. perimental setup and typical lattice parameters are described elsewhere [18].

Light propagating in weakly coupled, single mode waveguides, can be described by [19]:

$$
i \frac{n}{c} \frac{\partial A_{j}}{\partial t}=i \frac{\partial A_{j}}{\partial z}=\beta_{j} A_{j}+C_{j, j+1} A_{j+1}+C_{j, j-1} A_{j-1} .
$$

Here $A_{j}$ is the wave amplitude at site $j, \beta_{j}$ is the on-site eigenvalue, $C_{i, j}$ is the coupling constant or tunneling rate between two adjacent sites $i$ and $j$ (for a periodic lattice $C_{i, j} \equiv C$ is constant), and $z$ is the longitudinal space coordinate. The description by Eq. (2) is completely analogous to the quantum description of noninteracting electrons in a solid crystal in the tight-binding approximation, i.e., the discrete Schrödinger equation. The main differences are that (i) the spatial modulation of the index of refraction in the $x$ direction now plays the role of the tight-binding potential, and the $\beta_{j}$ s represent the propagation-constant eigenvalues of each waveguide in the lattice (ii) the evolution at a given time can be observed by measuring the intensity distribution at the corresponding position in the $z$ axis [11], since $z=c t / n$, where $c / n$ is the speed of light in the medium. The advantage of this system is the possibility to control the exact initial conditions for the light propagating inside the lattice. This is done by setting the width, the phase and the position across the lattice of the beam injected into the structure. In addition, this approach enables direct observation of the resulting wave function by measuring the distribution of light intensity at the sample's output [Fig. 1(b)]. Furthermore, the temporal evolution of the wave function can be observed by changing the sample length, or the initial conditions (e.g., $[13,20])$.

One of the hallmarks of QWs on ordered lattices is their ballistic propagation [2]. In order to observe this behavior, coherent light is injected into a single site in the lattice and the output intensity is measured. In Fig. 2 we compare the theoretical and the measured output distribution. The signature of ballistic propagation is clearly observed both at short and long propagation times [Fig. 2(a)]. Note that decoherence effects are negligible even after relatively long evolution in time, maintaining the detailed interference pattern predicted by theory [Fig. 2(b)]. Similar results, studied in a different context, were observed as early as in 1973 by Somekh et al. [21] on small scales in structures similar to the ones described above. The propagating photons tunnel from the origin site to an adjacent site, and immediately start tunneling to the next neighboring site. Through the tunneling between sites the photons accumulate a $\pi / 2$ phase, and an additional phase is accumulated continuously in each lattice site $j$, at a rate given by $\beta_{j}$. The interference of all these waves depends on the phase accumulated in each possible path, and gives rise to the observed intensity distribution. This description is practically identical to the description of the QW, where the light intensity corresponds to the probability distribu- 

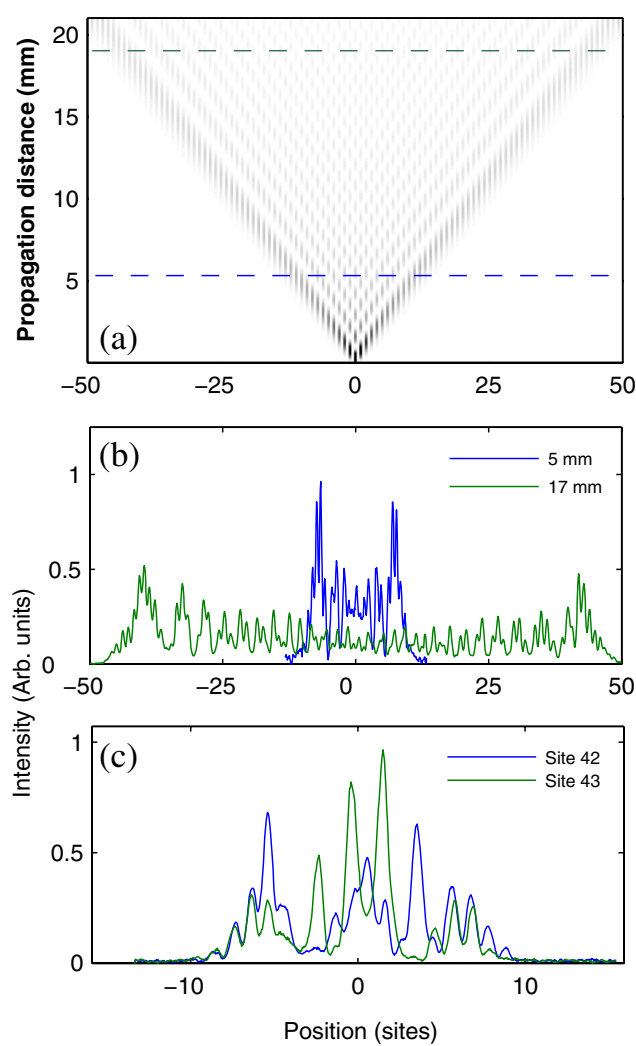

FIG. 2 (color online). (a) The theoretical prediction showing the ballistic evolution of the probability distribution of a CQW. The dashed lines correspond to the experimental measurements in (b). (b) The observed output pattern of light intensity after short (blue) and long (green) propagation in a periodic lattice. This well-known pattern is one of the hallmarks of the ballistic propagation of QWs. (c) Output patterns of light intensity resulting from injection of light into two adjacent single waveguides (sites 42 and 43) of a disordered lattice. The different patterns observed demonstrate the high sensitivity of the QW to the initial conditions in this case.

tion of the quantum particle. Since the single photon and many photon problems are described by the same probability distribution, experiments measuring light intensity are equivalent to performing a series of single photon experiments, from which the probability distribution is obtained. The propagation of more complex quantum states can be studied using correlated or entangled photons (see, for example, [22]). In this case the particle characteristics of the quantum walkers can be revealed by measuring two-photon correlation functions.

When disordered lattices are used [23,24], a different behavior is observed. Accumulated random phases of the random walker lead to destructive interferences that increase with distance from the origin. As a result, after a short ballistic propagation, the tails of the distribution are exponentially suppressed leaving the probability distribution exponentially localized to a small regime. This phenomena should be distinguished from a disordered related decoherence. Decoherence is related to temporal disorder, which induces a loss of phase coherence and results in a

transition into classical diffusion, characterized by an expanding Gaussian probability distribution $[25,26]$. Spatial disorder such as used here leads to an exponential (Anderson) localization (e.g., [27,28]), which is a coherent interference effect. In the context of CQWs, such behavior was found to be important for the efficiency of quantum algorithms [17,26,29].

QWs in disordered lattices are highly sensitive to the initial conditions. Figure 2(c) shows two output patterns of light intensity resulting from the injection of light into a single waveguide of a lattice and similar injection to an adjacent site of the same lattice. The different patterns observed demonstrate the high sensitivity of the QW to the exact initial conditions. This serves as a unique signature of the coherent nature of the $\mathrm{QW}$, which is not present in the classical case. In addition these results demonstrate the effect of disorder on QWs, where in this case the disorder was introduced through randomizing the tunneling rate between sites (off-diagonal disorder). The tails of the distribution still show the ballistic component of the regular QW. However, additional strong peaks now appear near the origin. At later times these peaks evolve (on average) into an exponentially localized distribution, while the ballistic side lobes are suppressed (see [24] for detailed discussion).

Several theoretical studies have been done on QWs with boundary conditions [30,31], that give rise to complex selfinterference patterns. In Fig. 3 we show experimental results of a QW with one reflecting boundary condition, compared with the theoretical analysis. A series of measurements is shown (horizontal cross sections), where in each measurement light was injected closer to the boundary. The observed pattern results from the self-interference of the incoming and reflected photons near the boundary, in agreement with theoretical predictions [30,32]. Although these are limited observations showing results of a short time propagation, longer waveguide lattices could be used

(a)

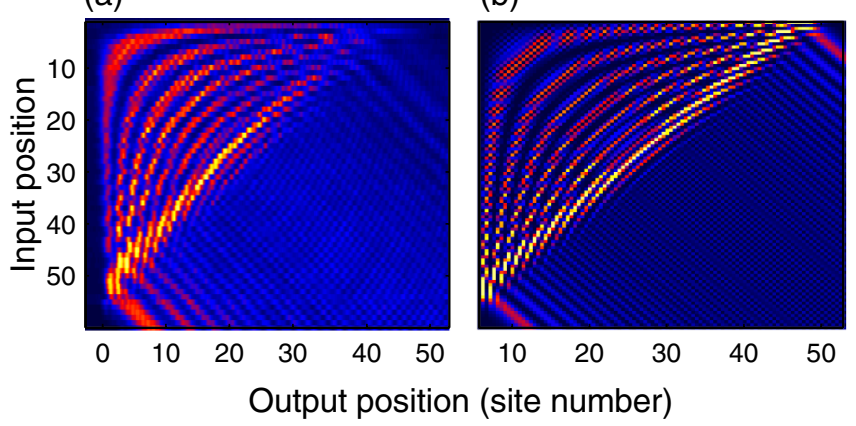

FIG. 3 (color online). (a) Measurements of the selfinterference patterns of QWs near a reflecting boundary. Horizontal cross sections show the left half of the probability distribution of the QW, at decreasing input site position (vertical axis), where position 0 marks the lattice left boundary. (b) Comparison to the theoretical analysis using the method of images [32]. 
to study the more complex evolution at later times. For example, such behavior of a two boundary conditions system can be used for studying quantum carpets containing fractal patterns $[12,29]$.

As an implementation of QWs, waveguide lattices carry some important advantages over other possible schemes. First, the technologies available for their fabrication or induction have reached a peak in recent years, enabling full control of every lattice parameters in one and two dimensions $[18,33]$, or limited yet real time control of lattice parameters in two dimensions [34]. Second, waveguide lattices have excellent structural stability; thus, in practice decoherence due to noise is negligible. The optical wavelength in our experiments (using AlGaAs wafers) is around $1.5 \mu \mathrm{m}$, the standard communication wavelength, and losses at these wavelengths are extremely small. This is highly important for quantum computational tasks where coherency is essential. Third, effects arising from the interactions between different random walkers in other possible implementations are eliminated here, due to the bosonic, noninteracting nature of photons.

In recent years, several quantum algorithms based on QWs have been suggested [35]. For realistic use of such algorithms one requires exponentially large systems. We note that as long as entanglement is not introduced, our system is limited to large but not exponentially large scale functionality. The lack of entanglement limits the number of the states of the system, which scales linearly with the number of waveguides. Our system, even without entanglement, can potentially implement QW algorithms, since quantum entanglement is not required for the algorithm implementation or its improved efficiency. Its only role in this case is to allow for a larger number of states (see, for example the discussion in [36]. Some of the suggested QW algorithms have been shown to provide polynomial or even exponential speed up [37,38]. Unfortunately, in all of the algorithms suggested so far the speed up of quantum over classical algorithms is achieved only when applied to high dimensional systems. Nevertheless, our system can still be used to implement and study these algorithms in lower dimensions.

In summary, we have demonstrated the strong correspondence between QWs and light propagation in waveguide lattices. This correspondence can be used to extend and interchange ideas and knowledge acquired in both fields (e.g., nonlinear behavior [11] in CQWs or entanglement effects $[39,40]$ in waveguide lattices). The high level of control, the accuracy, and the low decoherence rates achieved in waveguide lattices experiments provide a powerful tools for the study of QWs, and may prove useful in the implementation of QWs-based algorithms.

This work was supported by the German-Israeli Project Cooperation (DIP), NSERC and CIPI (Canada), and EPRSC (UK). Y. L. is supported by the Israeli Academy of Sciences and Humanities. H.P. and Y.L. thank the members of the WIS PIRATE club in which this study was initiated.

*hagai.perets@weizmann.ac.il

[1] A. Feynman and R.P. Hibbs, Quantum Mechanics and Path Integrals (McGraw-Hill, New-York, 1965).

[2] J. Kempe, Contemp. Phys. 44, 307 (2003).

[3] V. Kendon, Phil. Trans. R. Soc. A 364, 3407 (2006).

[4] S. Godoy and S. Fujita, J. Chem. Phys. 97, 5148 (1992).

[5] Y. Aharonov, L. Davidovich, and N. Zagury, Phys. Rev. A 48, 1687 (1993).

[6] E. Farhi and S. Gutmann, Phys. Rev. A 58, 915 (1998).

[7] B. Do et al., J. Opt. Soc. Am. B 22, 499 (2005).

[8] R. Côté et al., New J. Phys. 8, 156 (2006).

[9] O. Mülken et al., Phys. Rev. Lett. 99, 090601 (2007).

[10] J. Du et al., Phys. Rev. A 67, 042316 (2003).

[11] D. N. Christodoulides, F. Lederer, and Y. Silberberg, Nature (London) 424, 817 (2003).

[12] M. V. Berry, J. Phys. A 29, 6617 (1996).

[13] R. Iwanow et al., Phys. Rev. Lett. 95, 053902 (2005).

[14] R. Feynman, The Feynman Lectures on Physics (AddisonWesley, Boston, 1963), Vol. 3.

[15] N. W. Ashcroft and D. N. Mermin, Solid State Physics (Brooks Cole, Belmont, MA, 1976).

[16] O. Mülken and A. Blumen, Phys. Rev. E 71, 036128 (2005).

[17] J. P. Keating et al., Phys. Rev. A 76, 012315 (2007).

[18] H. S. Eisenberg et al., Phys. Rev. Lett. 81, 3383 (1998).

[19] A. Yariv, Quantum Electronics (Wiley, New-York, 1989).

[20] R. Morandotti et al., Phys. Rev. Lett. 83, 4756 (1999).

[21] S. Somekh et al., Appl. Phys. Lett. 22, 46 (1973).

[22] A. Politi et al., arXiv:0802.0136.

[23] T. Schwartz et al., Nature (London) 446, 52 (2007).

[24] Y. Lahini et al., Phys. Rev. Lett. 100, 013906 (2008).

[25] V. Kendon, Math. Struct. Comp. Sci. 17, No. 6, 1169 (2007).

[26] Y. Yin, D. E. Katsanos, and S. N. Evangelou, Phys. Rev. A 77, 022302 (2008).

[27] P. W. Anderson, Phys. Rev. 109, 1492 (1958).

[28] P. Sheng, Introduction to Wave Scattering, Localization, and Mesoscopic Phenomena (Springer, New-York, 1989).

[29] O. Mülken, V. Bierbaum, and A. Blumen, Phys. Rev. E 75, 031121 (2007).

[30] E. J. Amanatidis, D.E. Katsanos, and S. N. Evangelou, Phys. Rev. B 69, 195107 (2004).

[31] A. J. Bessen, arXiv:quant-ph/0609128.

[32] K. G. Makris and D. N. Christodoulides, Phys. Rev. E 73, 036616 (2006).

[33] A. Szameit et al., Opt. Express 13, 10552 (2005).

[34] J. W. Fleischer et al., Nature (London) 422, 147 (2003).

[35] A. Ambainis, arXiv:quant-ph/0403120.

[36] N. Bhattacharya, H. B. van Linden van den Heuvell, and R. J. Spreeuw, Phys. Rev. Lett. 88, 137901 (2002).

[37] A. M. Childs et al., in Proc. of STOC 2003 (2003), p. 59.

[38] A. M. Childs and J. Goldstone, Phys. Rev. A 70, 022314 (2004).

[39] I. Carneiro et al., New J. Phys. 7, 156 (2005).

[40] G. Abal et al., Phys. Rev. A 73, 042302 (2006). 